\title{
The impact of COVID-19 pandemic control on vaccine-preventable invasive bacterial diseases in Piedmont (Italy)
}

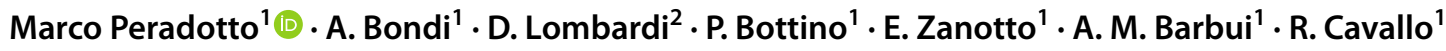

Received: 17 November 2021 / Accepted: 31 January 2022 / Published online: 16 February 2022

(c) The Author(s), under exclusive licence to Springer-Verlag GmbH Germany 2022

\begin{abstract}
Purpose The impact of SARS-CoV-2 pandemic on other pathogens is largely unknown. We aimed to compare the prevalence of vaccine-preventable invasive bacterial infections before and during the pandemic in Piedmont (Italy).

Methods We defined the monthly incidence of $S$. pneumoniae, H. influenzae and N. meningitides-invasive diseases from January 2010 to June 2021. Then, we compared the mean monthly cases during the previous 5 years (2015-2019) and the monthly cases in 2020 or 2021.

Results We found significant reductions for invasive pneumococcal diseases (IPDs) in adults and $H$. influenzae-invasive diseases in 2020 and 2021 in comparison to the previous years, but not for invasive meningococcal diseases and IPDs in children. Conclusions Further data are needed to confirm these findings and define possible post-pandemic evolutions in the epidemiology of vaccine-preventable invasive bacterial diseases.
\end{abstract}

Keywords Vaccine-preventable diseases $\cdot$ Bacterial-invasive infections $\cdot$ COVID-19 pandemic $\cdot$ Social distancing

\section{Introduction}

In Italy, vaccine-preventable invasive bacterial diseases (IBDs) are constantly under surveillance by regional and national organisations. This surveillance is essential to define the spread of these pathogens, to prevent local or national outbreaks. Moreover, it allows to monitor serotype or serogroup changes among populations, in relation to specific vaccine programmes [1].

Since February 2020, the COVID-19 pandemic has completely put under pressure the National Health Service in Italy, as well as in many other countries. Therefore, there was a reduction of its usual healthcare levels, ranging from emergencies not related to SARS-CoV-2 infection to all other routine activities. During the pandemic, several

Marco Peradotto

marcobio92@gmail.com

1 Microbiology and Virology Unit, University Hospital Città della Salute e della Scienza di Torino, C.so Bramante 88/90, 10126 Torino, Italy

2 Servizio di Riferimento Regionale di Epidemiologia per la Sorveglianza, la Prevenzione e il Controllo delle Malattie Infettive (SEREMI), Azienda Sanitaria Locale Alessandria, Alessandria, Italy countries registered a decrease in the incidence of community-acquired infections, such as influenza, respiratory syncytial virus, pertussis, varicella and invasive pneumococcal diseases (IPDs) [2, 3].

In Piedmont, the regional service of epidemiology (SeREMI), in cooperation with the regional reference laboratory at our university hospital, is responsible for the surveillance of vaccine-preventable IBDs. In particular, all cases of invasive disease caused by Streptococcus pneumoniae (SP), Haemophilus influenzae (HI) or Neisseria meningitidis (NM) are notified to SeREMI. Then, if possible, the bacterial isolate is sent to the regional reference laboratory for serotype or serogroup determination. Piedmont region, which represents approximately $7.5 \%$ of the whole Italian population, shows high vaccination rates in the paediatric population. In particular, vaccine coverage increased from $86 \%$ in 2010 to $92 \%$ in 2019 for pneumococcal conjugate vaccine 13 (PCV-13) at 24 months. In the same period, NM serogroup $\mathrm{C}$ vaccine coverage at 24 months grew from 71 to $91 \%$. Vaccine coverage for $\mathrm{HI}$ type $\mathrm{b}$ always reached at least $95 \%$ coverage at 24 months since 2010 [4].

The aim of this work was to describe the diffusion of IBDs from January 2010 to June 2021, with a focus on the impact of COVID-19 pandemic and all related control measures. In particular, Italy went to a complete lockdown 
in March and April 2020. After that, the second wave of COVID-19 imposed strict measures once again. A second time, from late October 2020 to late April 2021, Italian people movements and interactions were reduced to fight the "second wave" of COVID-19. Indeed, "basic measures" $(\mathrm{BM})$ are still applied: since the beginning of the pandemic, social distancing and frequent handwashing are strongly recommended, while face masks are mandatory at least in public indoors environment [5].

\section{Methods}

We defined the monthly cases of SP, HI and NM-invasive diseases, based on cases notification to SeREMI from January 2010 to June 2021. Data for SP were split, to differentiate the paediatric $(<15$ years old) and the adult population ( $>15$ years old). In fact, the availability of two different vaccines for children and adults, different vaccine coverage and the different SP prevalence in the two populations require a separate analysis. Data for SP in adults and HI were available from January 2012, when these surveillances started.

Student's $t$ test was performed to define variations between the mean monthly cases during the previous 5 years (2015-2019) and 2020 cases or the 2015-2019 mean monthly cases in the period January-June and the cases in the same period of 2021. Data were considered statistically different for $p<0.01$.

\section{Results}

SP in the paediatric population showed a general trend of decrease during the years, as shown in Fig. 1a. In 2020, we only registered two cases of IPDs in children, but compared to the mean cases of the previous 5 years we did not find statistical differences $(p=0.02)$. Both cases were caused by non-PCV-13 serotypes (19A and 23B), while during 2019, one case out of five was attributed to serotype 7F (PCV-13).

IPD cases in adults are shown in Fig. 1b. It is possible to observe an increase of cases from 2012 with a peak in 2017 (n. 297) and the classic seasonal peak during the winter. In 2020, only 94 cases were notified with a significant decrease compared to the previous years $(p=0.001)$. The same trend was observed during the first 6 months of 2021, when only 29 IPD cases were registered among adults. This data is significantly lower compared to the same period of the previous 5 years $(p=0.004)$ and significantly similar to the first 6 months of $2020(p=0.41)$. In this period, 73/94 IPD cases among adults were caused by non-PCV-13 serotypes (77.7\%), which is consistent with the general serotype switch we observed in the last 10 years (data not published). Interestingly, all other isolates were serotype 3 . Nonetheless, no significant differences were found in serotypes prevalence during the years. Considering the monthly cases in the period 2020-2021, the decrease in IPDs in comparison to the previous 5 years was evident (Fig. 1c). In particular, this decrease is more marked during the periods of more strict social distancing measures (SD) or lockdown (L), rather than periods of application of BM alone.

In the general low-prevalence setting of invasive meningococcal disease (IMD) in our region, we could observe a halving of cases in 2018, when we registered the lowest number of cases since 2010 (Fig. 1d). We could not define statistical differences in 2020 compared to the previous 5 years $(p=0.05)$. Based on typing, we observed a prevalence of serogroup B (Fig. 1e), with no significant differences between serogroups during years.

As regards $\mathrm{HI}$-invasive infections, before the pandemic we were registering a slight, but constant, increase in cases with a peak of 25 cases in 2019 (Fig. 1f). 57\% of isolates were unencapsulated non-typeable HI. During 2020, only six cases were reported, with a significant decrease in comparison to the previous 5 years $(p=0.004)$. In the same way, 2021 showed significant differences with the period 2015-2019 $(p=0.002)$ and significant similarity to 2020 $(p=0.21)$, even if the sample abundancy was very low.

\section{Discussion}

Since February 2020, the Italian healthcare system has been put under pressure by the COVID-19 pandemic. With the aim of reducing the spread of SARS-CoV-2, the Italian government applied strict social distancing measures, similarly to many other countries in the world. From the second wave (October 2020), these measures were differentiated based on regional epidemiology, thus avoiding a national lockdown [5]. In this perspective, we aimed to determine the impact of such measures on the diffusion of vaccine-preventable bacterial-invasive diseases in our region. In fact, SP, HI and NM-invasive infections share with SARS-CoV-2 and many other respiratory pathogens the need of close contact and droplets for their airborne transmission. Thus, social distancing and related protection measures have a great potential against all three pathogens, considering their efficacy against the high transmissibility of SARS-CoV-2 [6].

In general, we observed statistically significant decrease for IPDs among adults and HI-invasive diseases, both in 2020 and 2021. A decrease was registered also for IPDs in children and IMDs, but the few number of cases could not determine a statistical significance. The Italian Istituto Superiore di Sanità (ISS) in its 2020 report for IBDs highlighted the general decrease for all three pathogens in terms of cases notification [7]. In agreement with other authors $[2,3]$, the ISS takes into account the possibility of a partial 
$\mathbf{a}$

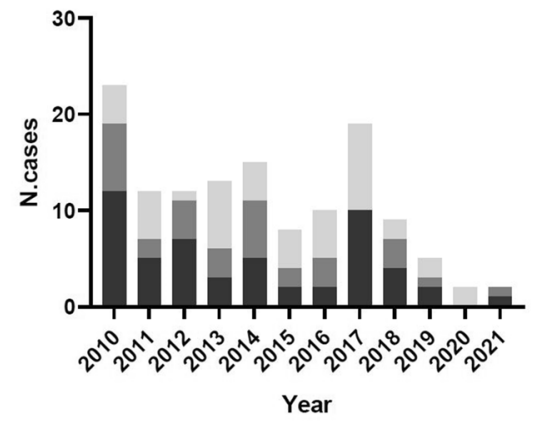

c

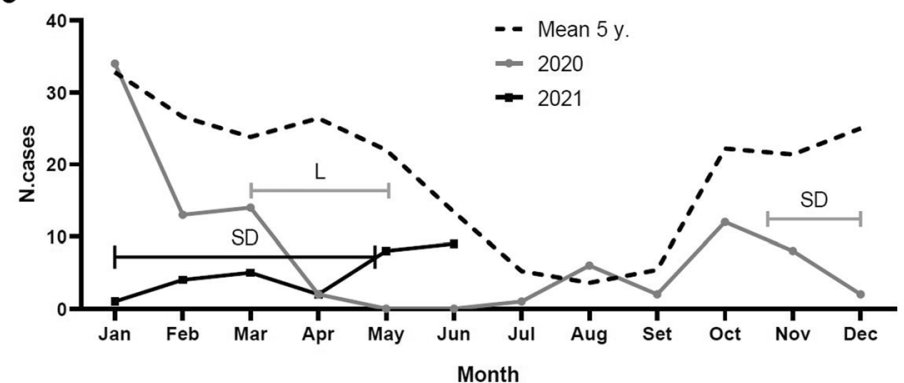

e

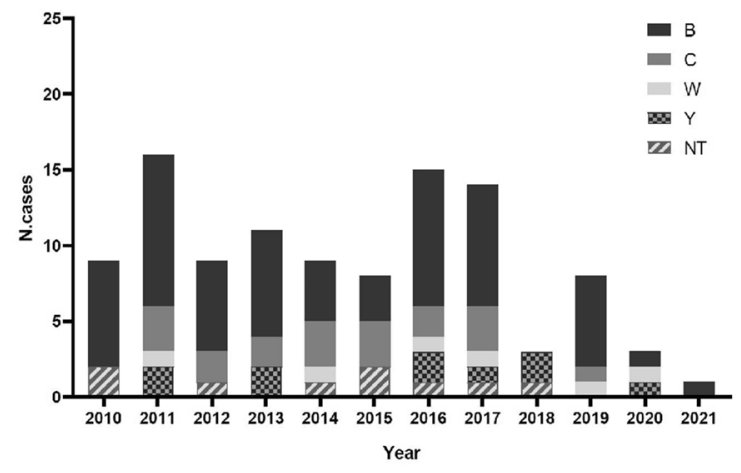

$\mathbf{b}$

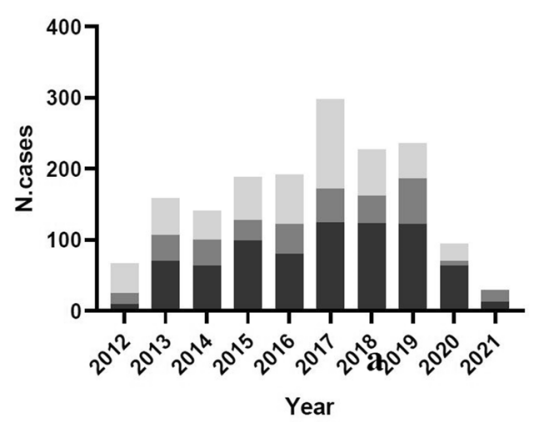

d

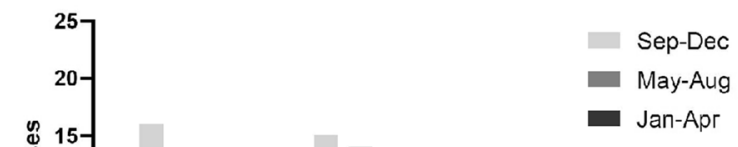

f

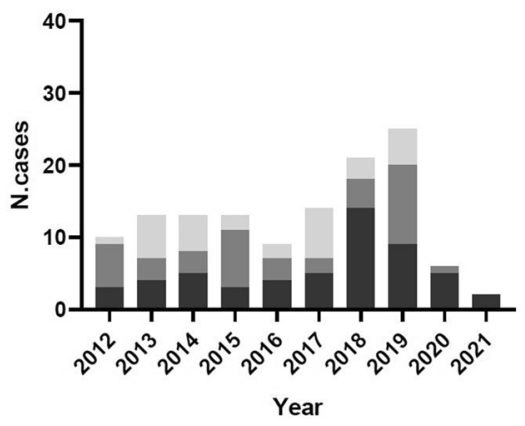

ary 2020; L: lockdown period; SD: more strict social distancing rules periods; $\mathbf{d}$ invasive meningococcal disease (IMD) cases notified from January 2010 to June 2021, based on 4-month periods; e IMD cases notified from January 2010 to June 2021, according to serogroup; NT non-typeable; f HI-invasive disease cases notified from January 2012 to June 2021, based on 4-month periods under-notification, especially in the more difficult periods of the pandemic. Indeed, the severity of IBDs itself cannot suggest a significant impact of under-diagnosis or undernotification coherent with such a reduction in IBDs [5, 7].

In general, IMDs strongly decreased in Italy and only 69 cases were registered in 2020, compared to 190 in 2019. In particular, the decrease started from March 2020, at the same time of national lockdown [5]. Due to the few number of cases, we could not determine monthly changes, but annual data clearly show the same decrease in the whole period March 2020-June 2021. Interestingly in France, the reduction of invasive meningococcal diseases (IMDs) was associated with an increase of respiratory manifestations due to non-hypervirulent Neisseria meningitidis in COVID-19 patients [8]. We could not highlight this phenomenon in our region and, to our knowledge, it was not reported in Italy.

Considering IPDs, we could determine a significant decrease only in the adult population. Older people are affected by lower vaccination rates compared to children, but they were more isolated thanks to lockdown or social distancing. In fact, in this population we could highlight the stronger decreases in IPDs coincidental to more strict social distancing rules periods. In the same way, in England, lockdown-related measures led to a strong reduction 
of invasive pneumococcal diseases (IPDs) in comparison to the same period of previous years [9].

Compared to IPDs and IMDs, there is lack of data about COVID-19 impact on the prevalence of $H$. influenzae-invasive diseases. Anyway, some studies reported $\mathrm{HI}$ as a major bacterial co-infection with COVID-19, in terms of both concomitant invasive infection and respiratory co-infection $[10,11]$. In our region, we could not determine an increase in HI-invasive infections, while respiratory infections lack a systematic surveillance. On the contrary, we determined a significant reduction of HI-invasive diseases in 2020 and 2021. As for IPDs in adults, the reasons stand in the impact of the pandemic on the adult population, more affected by this IBD and the prevalent airborne transmission of HI.

Finally, a recent model on the Japanese population hypothesized reduced vaccine coverage for the next years as a possible negative impact of the pandemic [12]. Unfortunately, regional data on 2020 vaccinations are still not available and this issue will require further investigations. Another limitation of this study regards its reduced coverage of the Italian population. Nonetheless, our findings are consistent with previous studies [1-5] and support the hypothesis of a decrease of vaccine-preventable IBDs due to COVID-19 spread and its consequences. In particular, different transmission mode based on pathogen or population age should be further investigated. For these reasons, additional local and national data will be needed to confirm our findings and to follow possible evolutions of vaccinepreventable IBDs prevalence after the pandemic.

\section{Funding None.}

\section{Declarations}

Conflict of interest The authors declare that they have no conflict of interest.

\section{References}

1. Pezzotti P, Bellino S, Riccardo F, Lucaroni F, Cerquetti M, Pantosti A, Rezza G, Stefanelli P. Vaccine preventable invasive bacterial diseases in Italy: a comparison between the national surveillance system and recorded hospitalizations, 2007-2016. Vaccine. 2019;37:41-8. https://doi.org/10.1016/j.vaccine.2018. 11.047 (Epub 2018 Nov 23. PMID: 30478004)
2. Chan KS, Liang FW, Tang HJ, Toh HS, Yu WL. Collateral benefits on other respiratory infections during fighting COVID-19. Med Clin (Barc). 2020;155:249-53. https://doi.org/10.1016/j.medcli. 2020.05.026 (PMID: 32586667; PMCID: PMC7274613).

3. Yun HE, Ryu BY, Choe YJ. Impact of social distancing on incidence of vaccine-preventable diseases. South Korea J Med Virol. 2021;93:1814-6. https://doi.org/10.1002/jmv.26614 (Epub 2020 Nov 1. PMID: 33079384).

4. Servizio di riferimento Regionale di Epidemiologia per la sorveglianza, la prevenzione e il controllo delle Malattie Infettive (SEREMI). Malattie prevenibili da vaccino. SeREMI, 2021. https:// www.seremi.it/sites/default/files/REPORT\%20MABI\%202020\% 20ed\%202021.pdf

5. Stefanelli P, Fazio C, Vacca P, Neri A, Ambrosio L, Rezza G. Did social distancing measures deployed for SARS-CoV-2/COVID-19 control have an impact on invasive meningococcal disease? Pathog Glob Health. 2021. https://doi.org/10.1080/20477724.2021. 1995657 (Epub ahead of print. PMID: 34844520).

6. Rabaan AA, Al-Ahmed SH, Haque S, Sah R, Tiwari R, Malik YS, Dhama K, Yatoo MI, Bonilla-Aldana DK, Rodriguez-Morales AJ. SARS-CoV-2, SARS-CoV, and MERS-COV: a comparative overview. Infez Med. 2020;28:174-84 (Ahead of print. PMID: 32275259).

7. Istituto Superiore di Sanità, Sorveglianza delle Malattie Batteriche Invasive in Italia. Rapporto Consolidato 2019. https://www.iss.it/ sn-mbi-rapporti-iss

8. Taha MK, Deghmane AE. Impact of COVID-19 pandemic and the lockdown on invasive meningococcal disease. BMC Res Notes. 2020;13:399. https://doi.org/10.1186/s13104-020-052419 (PMID: 32854773; PMCID: PMC7450896).

9. Amin-Chowdhury Z, Aiano F, Mensah A, Sheppard CL, Litt D, Fry NK, Andrews N, Ramsay ME, Ladhani SN. Impact of the coronavirus disease 2019 (COVID-19) pandemic on invasive pneumococcal disease and risk of pneumococcal coinfection with severe acute respiratory syndrome coronavirus 2 (SARS-CoV-2): prospective national cohort study. England Clin Infect Dis. 2021;72:e65-75. https://doi.org/10.1093/cid/ciaa1728 (PMID: 33196783; PMCID: PMC7717180).

10. Zhu X, Ge Y, Wu T, Zhao K, Chen Y, Wu B, Zhu F, Zhu B, Cui L. Co-infection with respiratory pathogens among COVID-2019 cases. Virus Res. 2020;285: 198005. https://doi.org/10.1016/j. virusres.2020.198005 (Epub 2020 May 11. PMID: 32408156; PMCID: PMC7213959).

11. Lansbury L, Lim B, Baskaran V, Lim WS. Co-infections in people with COVID-19: a systematic review and meta-analysis. J Infect. 2020;81:266-75. https://doi.org/10.1016/j.jinf.2020. 05.046 (Epub 2020 May 27. PMID: 32473235; PMCID: PMC7255350).

12. Kitano T, Aoki H. A model for the incremental burden of invasive Haemophilus influenzae type $b$ due to a decline of childhood vaccination during the COVID-19 outbreak: a dynamic transmission model in Japan. Vaccine. 2021;39:343-9. https://doi. org/10.1016/j.vaccine.2020.11.053 (Epub 2020 Nov 24 PMID: 33280853). 